\title{
Osteotomy of the greater trochanter: effect on gluteus medius function
}

\author{
Martin Beck $^{1,2} \cdot$ A. Krüger ${ }^{2}$ C. Katthagen ${ }^{3} \cdot$ S. Kohl ${ }^{2}$
}

Received: 18 June 2014/ Accepted: 20 March 2015/Published online: 1 April 2015

(C) Springer-Verlag France 2015

\begin{abstract}
Purpose Advancement of the greater trochanter alters the function of the gluteus medius muscle. However, with the exception of clinical studies and biomechanical lever arm studies, no publications that analyze the consequences of advancement of the greater trochanter on the muscle function exist. The aim of the study was to analyze the mechanical changes of gluteus medius after osteotomy of the greater trochanter in a lab setting.

Methods An anatomical study of origin and insertion of the gluteus medius was carried out on four hips. Based on the dissections, a string model was developed dividing the muscle into five sectors. Changes in muscle fiber length were measured for every $10^{\circ}$ of flexion, internal and external rotation and abduction with the trochanter in anatomic, proximalized and distalized positions.

Results Distalization of the trochanter leads to an imbalance of muscle action, moving the isometric sector of the muscle anteriorly with more muscle sectors being active during flexion and less during extension. Stretching of the muscle increases passive forces but decreases the force generation capacity of the muscle and at the same time increased muscle fiber excursion may require more energy consumption, which may explain earlier fatigue of the
\end{abstract}

Martin Beck

martin.beck@luks.ch

1 Clinic for Orthopedic and Trauma Surgery, Luzerner Kantonsspital, 6004 Lucerne, Switzerland

2 Department of Orthopedic Surgery, University of Bern, Inselspital, 3010 Bern, Switzerland

3 Department of Trauma and Reconstructive Surgery, Diakoniekrankenhaus Friederikenstift $\mathrm{GmbH}$, 30169 Hannover, Germany abductor musculature after distalization of the trochanter. For abduction, distalization of the muscle attachment leads to a change in contraction pattern from isometric to isotonic. Optimal balancing and excursion of the muscle is when the tip of the greater trochanter is at level with the hip rotation center.

Conclusions In hips with high riding trochanter, the optimal position is at the level of the center of hip rotation. Excessive distalization should be avoided. As the conclusions and considerations are based on a lab setting, transfer to clinical practice may not necessarily apply.

Keywords Abductor function - Osteotomy greater trochanter $\cdot$ Muscle function $\cdot$ Hip

\section{Introduction}

Advancement of the greater trochanter alters mechanics and function of the gluteus medius muscle and is recommended to improve abductor function for pediatric and adolescent hip pathologies, including Perthes' disease, coxa vara, and slipped femoral capital epiphysis $[13,17]$. It yields satisfactory results with improved abductor strength in the majority of cases $[1,15,17,18,24]$. With the popularization of joint preserving surgery of the hip by means of a trochanteric flip approach [6, 11], distal advancement of the greater trochanter is recommended as an adjunct to treat femoro-acetabular impingement in certain pathologies $[1,12,16]$. Some biomechanical studies focus on the theoretical advantage of the change to the lever arms $[10,15,24]$, but the effect of the changes in muscle fiber length and of the transposition of the trochanter on function of the gluteus medius muscle has not yet been investigated. According to the current knowledge, an extensile literature 
research did not reveal any publications on the biomechanics of the gluteus medius muscle after transposition of the greater trochanter.

The motivation for this study was our clinical observation that, contrary to our expectations, patients with distalization of the greater trochanter often complained of pain and fatigue in the abductor musculature, which could not be explained with current knowledge. We therefore sought to quantify the change in the biomechanics of the gluteus medius muscle after transposition of the osteotomized greater trochanter, and to identify factors that may explain the fatigue of the abductor musculature after advancement.

\section{Materials and methods}

In order to verify current knowledge of origin and insertion of the gluteus medius, its anatomy was studied in four hips in three fresh bodies. The age was between 60 and 80 years of age and none of them had previous surgery around the hip. At dissection, the hips looked normal, without signs of deformity or joint degeneration. Based on the anatomical findings, a string model was developed that allowed to record the change of the muscle fiber length during motion as was described previously [2].

For dissection, the bodies were placed in a lateral decubitus position. A longitudinal incision was made from the iliac crest to the mid-thigh, and the skin and subcutaneous tissue dissected off the fascia covering the abductor musculature. The fascia over the gluteus maximus and gluteus medius was incised in the interval between the two muscles and the gluteus maximus was separated from the gluteus medius and detached from the ilium. Anteriorly, the tensor fascia latae muscle was identified and separated from the gluteus medius together with the iliotibial band. The remaining fascia was then removed, except the part adherent to the gluteus medius muscle, the size and shape of which was recorded. The gluteus medius was subsequently dissected off the gluteus minimus and the bony origin was recorded alongside the insertion on the greater trochanter.

Based on the anatomic findings, a string model of the gluteus medius was developed [2], using synthetic bones (Sawbones ${ }^{\circledR}$ ) of a right hemipelvis and a right proximal femur, its size corresponding approximately to a standard sized human (Fig. 1). The hemipelvis was fixed with screws to a board. Because the rough surface of the femoral head did not allow smooth movement, a total hip prosthesis was implanted to allow reproducible motion of the hip joint. There were no soft tissues to be balanced. The shaft of the prosthesis was implanted such that the tip of the greater trochanter was at the level of the center of rotation, where it has been reported to be in the normal hip [23].
The muscle area was divided into five sectors, sector 1 being most anterior and sector 5 most posterior. Each sector of the muscle was represented by a 2 -mm thick cord, which was firmly fixed to its insertion on the greater trochanter and directed through pulleys at the center of the origin of each sector. The cords were then further routed to a scale, where the excursion of each cord was measured independently. Millimeter paper on the surface of the board was used as a scale unit. The ends were fixed to an elastic band to maintain tension.

A gravity inclinometer (goniometer) was fixed to the distal femur. Rotation and abduction/adduction was directly measured with the inclinometer. For flexion/extension the gravity inclinometer was used to control rotation and an additional goniometer to measure flexion. Measurement in each direction began with the hip in neutral position. Before the first run, the cords were calibrated at a neutral position of $0^{\circ}$ flexion, $0^{\circ}$ abduction, and $0^{\circ}$ rotation, from where all measurements began. Measurements were taken in $10^{\circ}$ increments from $20^{\circ}$ extension to $90^{\circ}$ flexion. Additional measurements were performed in $10^{\circ}$ increments from $20^{\circ}$ abduction to $20^{\circ}$ adduction, and from $20^{\circ}$ internal rotation to $20^{\circ}$ external rotation. Three measurements were taken for each movement and an average was calculated. The greater trochanter was then osteotomized and reattached with two screws. Measurements were repeated with the trochanter reattached in a $1 \mathrm{~cm}$ proximalized and $1 \mathrm{~cm}$ distalized position. The starting points of the measurements were calibrated before transposition in order to obtain absolute values for each movement for the repositioned trochanter, including lengthening or shortening in the new position. Later, for comparison of the graphs, the values at the neutral positions were calculated to zero. Muscle excursions for the advanced trochanter were measured and presented as a percentage of the original length of the muscle sectors. The intraobserver kappa value of the measurements was calculated with the accuracy of measurement set at $1 \mathrm{~mm}$.

Changing the position of the trochanter in relation to the center of rotation also changes the lever arm in the sagittal plane. To measure this change, the insertions of sectors one, three and five were marked with 3-mm colored pinheads. The length of the lever arm was measured for the normal, distalized, and proximalized trochanters, and compared for $0^{\circ}, 30^{\circ}$, and $60^{\circ}$ of flexion. To measure a calibrated photograph was taken at each position. Keeping the center of rotation at an identical position, the three photographs were superimposed digitally and the change of lever arms was measured in relation to an axis through the center of rotation. To represent the fan-shaped orientation of the muscle fibers, the axis for the central muscle fibers (sector 3) was perpendicular, the axis for the posterior sector 5 inclined $20^{\circ}$ posteriorly and for sector $120^{\circ}$ anteriorly. 


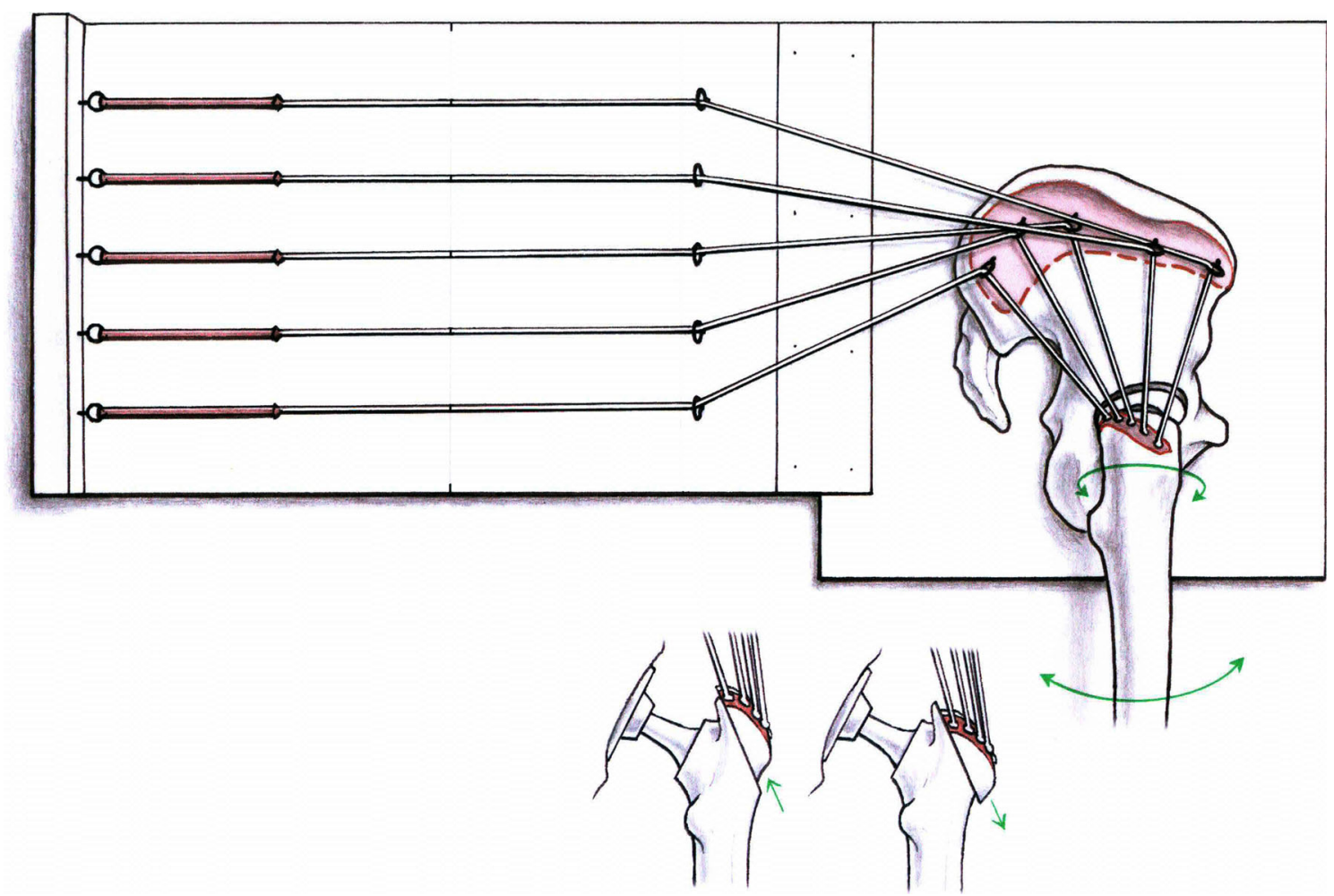

Fig. 1 Schematic of the string model

\section{Results}

\section{Anatomical dissection}

The gluteus medius is a strong fan-shaped muscle with a bony and fascial origin. Both origins are wedge-shaped, with the base of the bony origin posterior and the base of the fascial origin anterior. The combination of the two origins results in an evenly sized area throughout the entire width of the muscle (Fig. 2a, b). From both origins, the fibers merge into a central tendon that is longer and better visible posteriorly. The most anterior fibers insert directly onto the greater trochanter. The insertion on the greater trochanter starts at the posterosuperior facet and continues obliquely down the lateral facet, ending at the lateral border of the anterior facet. Muscle fiber length was approximately $10 \mathrm{~cm}$ in all parts of the muscle.

\section{Simulated hip flexion}

The data of the averaged absolute measurements are given in Table 1. Table 2 shows the values calculated to 0 at the neutral position. During flexion of the hip, the two anterior sectors shorten, the length of the central sector remains isometric and the two posterior sectors lengthen (Fig. 3a). The excursions of the anterior and posterior sectors are balanced around the central sector. Transfer of the greater trochanter in either direction disturbs the balance. After distalization of the trochanter, the isometric sector moves anteriorly (Fig. 3b) resulting in less active muscle sectors for flexion but more for extension. After proximalization of the trochanter, the opposite phenomenon is observed (Fig. 3c).

There is not only an imbalance in active muscle sectors, but also a change in the excursion itself. Transposition of the greater trochanter had the greatest effect on the muscle fibers of sector 1. Distalization of the trochanter led to an increase of muscle excursion of $13 \%$ at $70^{\circ}$ (Table 3). At $90^{\circ}$ of flexion, the muscle excursion increased $45 \%$. Proximalization of the trochanter led to a decrease in muscle excursion of $72 \%$ at $90^{\circ}$ of flexion. In sector 3 , proximalization of the trochanter caused inversion and shortening of the excursion and in sector 5 , the excursion increased by $13 \%$ at $70^{\circ}$ and $32 \%$ at $90^{\circ}$ of flexion.

During walking, the hip moves from about $30^{\circ}$ of flexion at heel strike to $10^{\circ}$ of extension at toe lift. During this, sectors 4 and 5 are active and shorten by 8.3 and $12.3 \mathrm{~mm}$, respectively. With the distalized trochanter, the excursion for sector 4 was $10 \mathrm{~mm}$, and for sector 5 was $14.3 \mathrm{~mm}$. The increase in excursion was $20 \%$ for sector 4 and $16 \%$ for sector 5 . The increase would be higher for climbing stairs, where hip flexion may reach $70^{\circ}$ and active extension of 
the hip from $70^{\circ}$ to $0^{\circ}$ is necessary. The excursion for sectors 4 and 5-the most active muscle sectors when extending a flexed hip-increased in sector 4 by $27 \%$ for
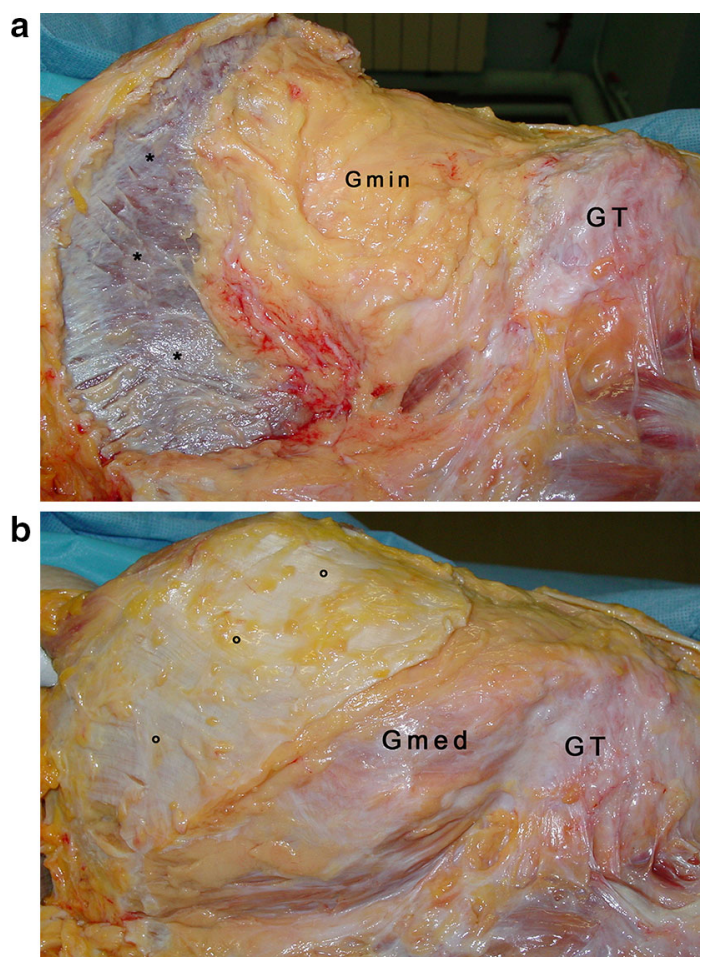

Fig. 2 a Area of origin of the gluteus medius muscle. The bony origin (asterisks) from the iliac wing is shown, with the base of the wedge being posteriorly. GT greater trochanter, Gmin M. gluteus minimus (fatty degeneration). b The fascial origin (circles) of the muscle with the base of the wedge lying anterior. The combination of the two origins results in an even area of origin from anterior to posterior. GT greater trochanter, Gmed M. gluteus medius the proximalized and $24 \%$ for the distalized trochanter but in sector 5 , it decreased by $3 \%$ for the proximalized and increased by $17 \%$ for the distalized trochanter.

\section{Simulated hip abduction/adduction}

The excursion for abduction of up to $20^{\circ}$ was the same for the three anterior sectors $(-12,-13$, and $-13 \mathrm{~mm})$. The two posterior sectors showed less excursion $(-8.5$ and $-6.5 \mathrm{~mm}$ ). Proximalization of the trochanter led to an increase in excursion of $23-25 \%$ for the 3 anterior sectors, and of $53-70 \%$ for the posterior sectors. Distalization caused an increase of excursion of 67-70\% for the anterior 3 sectors and $100-115 \%$ for the 2 posterior sectors.

\section{Simulated hip rotation}

The excursions of the sectors were divergent for all sectors (Fig. 4). Starting from the neutral position, only the fibers of sector 1 shortened during internal rotation. During external rotation, the three posterior sectors shortened. Interestingly, sector 2 elongated during internal and external rotation. The course of the sectors was not influenced by the position of the trochanter, and remained unchanged except for the starting position on the vertical axis.

\section{Change of lever arm}

Transposition of the greater trochanter also affected the lever arms in the sagittal plane (Fig. 5a-c; Table 4). The lever for sector 1 increased on average by $69 \%$ for all three positions for the distalized trochanter and decreased by $35 \%$ for the proximalized trochanter.

Table 1 Absolute values of the muscle excursion of sector $1-5$ in $\mathrm{mm}$, for flexion from $-20^{\circ}$ to $90^{\circ}$ with the trochanter at its anatomic (N), proximalized (P), and distalized (D) position

\begin{tabular}{|c|c|c|c|c|c|c|c|c|c|c|c|c|c|c|c|}
\hline \multirow[t]{2}{*}{ Flexion } & \multicolumn{3}{|l|}{$\mathrm{S} 1$} & \multicolumn{3}{|l|}{$\mathrm{S} 2$} & \multicolumn{3}{|l|}{ S3 } & \multicolumn{3}{|l|}{ S4 } & \multicolumn{3}{|l|}{ S5 } \\
\hline & $\mathrm{N}$ & $\mathrm{P}$ & $\mathrm{D}$ & $\mathrm{N}$ & $\mathrm{P}$ & D & $\mathrm{N}$ & $\mathrm{P}$ & $\mathrm{D}$ & $\mathrm{N}$ & $\mathrm{P}$ & D & $\mathrm{N}$ & $\mathrm{P}$ & $\mathrm{D}$ \\
\hline-20.0 & 4.0 & -5.0 & 16.0 & 1.0 & -8.0 & 12.0 & -1.0 & -10.0 & 9.0 & -3.7 & -13.0 & 6.0 & -4.7 & -13.0 & 4.0 \\
\hline-10.0 & 3.0 & -6.0 & 14.0 & 0.7 & -8.0 & 12.3 & -1.0 & -9.0 & 10.0 & -3.0 & -11.0 & 9.0 & -4.0 & -12.0 & 7.7 \\
\hline 0.0 & 0.0 & -8.0 & 12.3 & 0.0 & -8.0 & 12.3 & 0.0 & -9.0 & 12.0 & 0.0 & -10.0 & 12.0 & 0.0 & -10.0 & 11.0 \\
\hline 10.0 & -2.7 & -10.0 & 9.3 & -0.3 & -9.0 & 12.3 & 1.0 & -9.0 & 13.3 & 2.3 & -9.0 & 15.0 & 3.3 & -8.0 & 15.0 \\
\hline 20.0 & -6.3 & -11.0 & 5.7 & -1.7 & -9.0 & 12.0 & 1.0 & -8.0 & 15.0 & 4.0 & -7.0 & 17.7 & 6.3 & -6.0 & 18.7 \\
\hline 30.0 & -11.0 & -12.0 & 2.0 & -4.0 & -9.0 & 10.7 & 0.7 & -8.0 & 14.7 & 5.3 & -5.0 & 19.0 & 8.3 & -3.0 & 22.0 \\
\hline 40.0 & -14.3 & -13.0 & -2.3 & -5.3 & -8.0 & 8.7 & 0.0 & -6.0 & 14.7 & 7.0 & -2.0 & 20.7 & 11.0 & 0.0 & 23.3 \\
\hline 50.0 & -17.3 & -14.0 & -7.0 & -7.3 & -8.0 & 6.0 & -0.3 & -4.0 & 14.0 & 7.7 & 1.7 & 21.0 & 13.3 & 3.3 & 26.0 \\
\hline 60.0 & -21.3 & -15.0 & -11.0 & -10.0 & -7.0 & 3.0 & -1.7 & -3.0 & 13.0 & 8.3 & 3.0 & 22.3 & 14.3 & 7.0 & 27.7 \\
\hline 70.0 & -24.3 & -16.0 & -15.3 & -12.0 & -7.0 & 0.7 & -2.7 & -2.0 & 11.7 & 9.3 & 7.0 & 22.0 & 17.7 & 10.0 & 29.0 \\
\hline 80.0 & -24.0 & -14.0 & -19.0 & -13.0 & -6.0 & -3.0 & -3.0 & 1.0 & 10.7 & 10.3 & 10.0 & 22.7 & 17.7 & 13.0 & 30.0 \\
\hline 90.0 & -24.3 & -15.0 & -23.0 & -13.7 & -5.3 & -6.3 & -3.0 & 1.0 & 8.7 & 12.0 & 12.0 & 22.0 & 19.7 & 16.0 & 30.0 \\
\hline
\end{tabular}


Table 2 Values of the muscle excursion normalized to the 0 position of sector $1-5$ in mm, for flexion from $-20^{\circ}$ to $90^{\circ}$ with the trochanter at its anatomic $(\mathrm{N})$, proximalized $(\mathrm{P})$, and distalized $(\mathrm{D})$ position

\begin{tabular}{|c|c|c|c|c|c|c|c|c|c|c|c|c|c|c|c|}
\hline \multirow[t]{2}{*}{ Flexion } & \multicolumn{3}{|l|}{$\mathrm{S} 1$} & \multicolumn{3}{|l|}{$\mathrm{S} 2$} & \multicolumn{3}{|l|}{ S3 } & \multicolumn{3}{|l|}{ S4 } & \multicolumn{3}{|l|}{ S5 } \\
\hline & $\mathrm{N}$ & $\mathrm{P}$ & $\mathrm{D}$ & $\mathrm{N}$ & $\mathrm{P}$ & $\mathrm{D}$ & $\mathrm{N}$ & $\mathrm{P}$ & $\mathrm{D}$ & $\mathrm{N}$ & $\mathrm{P}$ & $\mathrm{D}$ & $\mathrm{N}$ & $\mathrm{P}$ & $\mathrm{D}$ \\
\hline-20.0 & 4.0 & 3.0 & 3.7 & 1.0 & 0.0 & -0.3 & -1.0 & -1.0 & -3.0 & -3.7 & -13.0 & -6.0 & -4.7 & -3.0 & -7.0 \\
\hline-10.0 & 3.0 & 2.0 & 1.7 & 0.7 & 0.0 & 0.0 & -1.0 & 0.0 & -2.0 & -3.0 & -1.0 & -3.0 & -4.0 & -2.0 & -3.3 \\
\hline 0.0 & 0.0 & 0.0 & 0.0 & 0.0 & 0.0 & 0.0 & 0.0 & 0.0 & 0.0 & 0.0 & 0.0 & 0.0 & 0.0 & 0.0 & 0.0 \\
\hline 10.0 & -2.7 & -2.0 & -3.0 & -0.3 & -1.0 & -1.6 & 1.0 & 0.0 & 1.3 & 2.3 & 1.0 & 3.0 & 3.3 & 2.0 & 4.0 \\
\hline 20.0 & -6.3 & -3.0 & -6.6 & -1.7 & -1.0 & -0.3 & 1.0 & 0.0 & 3.0 & 4.0 & 3.0 & 5.7 & 6.3 & 4.0 & 7.7 \\
\hline 30.0 & -11.0 & -4.0 & -10.3 & -4.0 & -1.0 & -1.6 & 0.7 & 1.0 & 2.7 & 5.3 & 5.0 & 7.0 & 8.3 & 7.0 & 11.0 \\
\hline 40.0 & -14.3 & -5.0 & -14.6 & -5.3 & 0.0 & -3.6 & 0.0 & 3.0 & 2.7 & 7.0 & 8.0 & 8.7 & 11.0 & 10.0 & 12.3 \\
\hline 50.0 & -17.3 & -6.0 & -19.3 & -7.3 & 0.0 & -6.3 & -0.3 & 5.0 & 2.0 & 7.7 & 11.7 & 9.0 & 13.3 & 13.3 & 15.0 \\
\hline 60.0 & -21.3 & -7.0 & -23.3 & -10.0 & 1.0 & -9.3 & -1.7 & 6.0 & 1.0 & 8.3 & 13.0 & 10.3 & 14.3 & 17.0 & 16.7 \\
\hline 70.0 & -24.3 & -8.0 & -27.6 & -12.0 & 1.0 & -11.6 & -2.7 & 7.0 & -0.3 & 9.3 & 17.0 & 10.0 & 17.7 & 20.0 & 18.0 \\
\hline 80.0 & -24.0 & -6.0 & -31.3 & -13.0 & 20. & -15.3 & -3.0 & 10.0 & -1.3 & 10.3 & 20.0 & 10.7 & 17.7 & 23.0 & 19.0 \\
\hline 90.0 & -24.3 & -7.0 & -35.3 & -13.7 & 2.7 & -18.6 & -3.0 & 10.0 & -3.3 & 12.0 & 22.0 & 10.0 & 19.7 & 26.0 & 19.0 \\
\hline
\end{tabular}

For sector 5, distalization lead to a decrease of the lever by approximately $10 \%$, and the lever arm inverted and moved to the other side of the action line at $60^{\circ}$ of flexion. Proximalization led to an average increase of the lever arm by $31 \%$ at all $3^{\circ}$ of flexion.

For the central sector, distalization led to an increase of lever arm (190\%) and to high excursion of the insertion. Proximalization did not change the lever arms significantly, while the excursion of the insertion decreased.

\section{Intraobserver variability}

The kappa value for intraobserver reliability was 0.70 , which is good.

\section{Discussion}

Our anatomical dissections revealed that the gluteus medius originates from both the iliac wing and a strong fascia overlying the gluteus medius. The muscle fibers from both origins converge and blend into a central tendon. The fascial origin of gluteus medius was described previously and named fascia glutea [19]. In addition, the presence of the two origins has been described previously [25] but in more recent anatomical descriptions the fascial origin often is omitted $[3,20]$. A recent study again looked in detail at the anatomy of gluteus medius and again described the origin form the gluteal fascia [8]. Looking at the bony origin alone, which commonly is done, one would conclude that the posterior part of gluteus medius is much stronger than the anterior part. However, considering the fascial origin, the muscle has an even area of origin from posterior to anterior (Fig. 2a, b). The strength generated by the muscle therefore should not differ substantially between the various sectors used for this study. Furthermore, from a clinical point of view, all parts are equally important. The division of gluteus medius into five sectors follows previous descriptions [8]. The presence of the central tendon also explains the shape of the posterior border, where it appears as if there was an additional muscle belly. This is, in reality, the medial part of the muscle that arises from the iliac wing and blends into the central tendon, which is corroborated by the recent study of Flack et al. [8].

Distal advancement of the greater trochanter was originally recommended to increase the stability of a total hip replacement $[5,7,14]$ and is currently used to improve abductor function for pediatric hip pathologies [13, 17] and as an adjunct in the treatment of femoro-acetabular impingement in certain pathologies $[1,12,16]$. However, it was questioned whether the advancement improved muscle function by increasing tension in the muscle and placing the muscle in a more advantageous position of the lengthtension curve [22].

From the current study, we conclude that the optimal position of the greater trochanter, with regard to muscle excursion of the various sectors and lever arms, is with the tip of the greater trochanter at the level of the center of rotation of the femoral head. This is supported by radiographic and clinical studies [15, 23]. At this position, the excursions of the five muscle sectors are symmetrically balanced around the central isometric sector (sector 3 ) for motion in the sagittal plane. During flexion, the two anterior sectors shorten and the two posterior sectors lengthen. This means that the anterior part of gluteus medius is active during flexion, and the posterior part is active during 
Fig. 3 a Overview of the muscle fiber excursion of sector $1-5$ with the anatomically positioned trochanter. Negative values on the $y$ axis correspond to shortening of the muscle sector. b Overview of the muscle fibers excursion of sector 1-5 with the trochanter distalized by $1 \mathrm{~cm}$. c Overview of the muscle fibers excursion of sector $1-5$ with the trochanter proximalized by $1 \mathrm{~cm}$ a

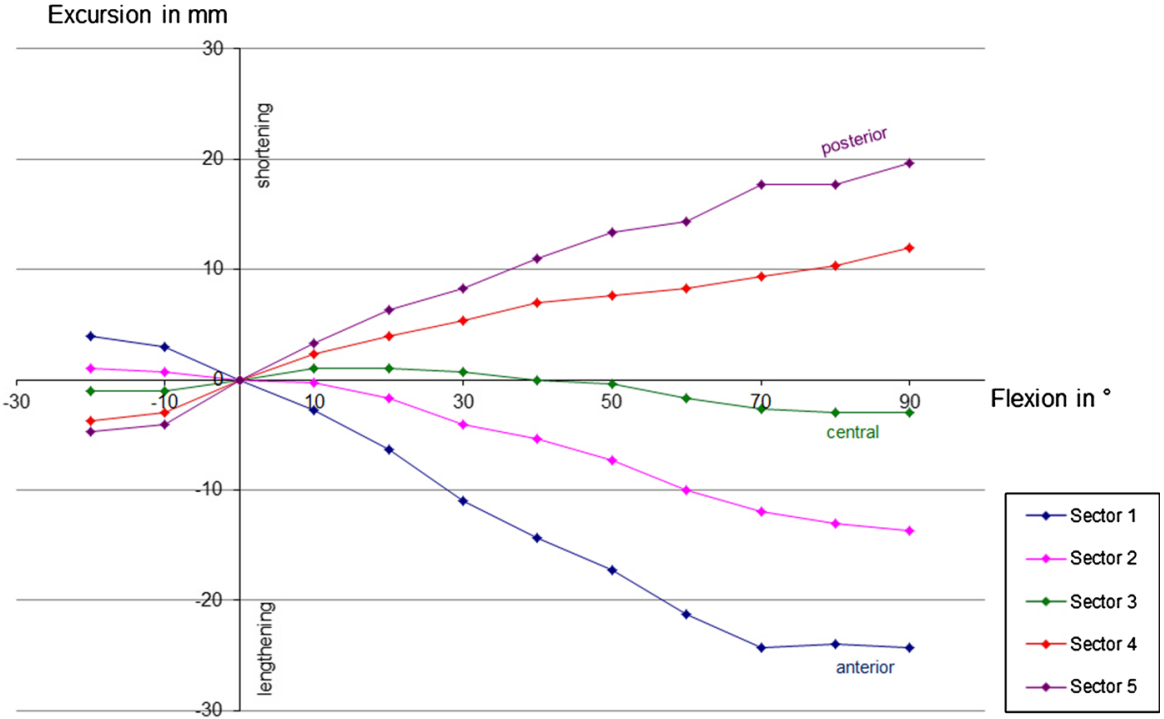

b Excursion in $\mathrm{mm}$

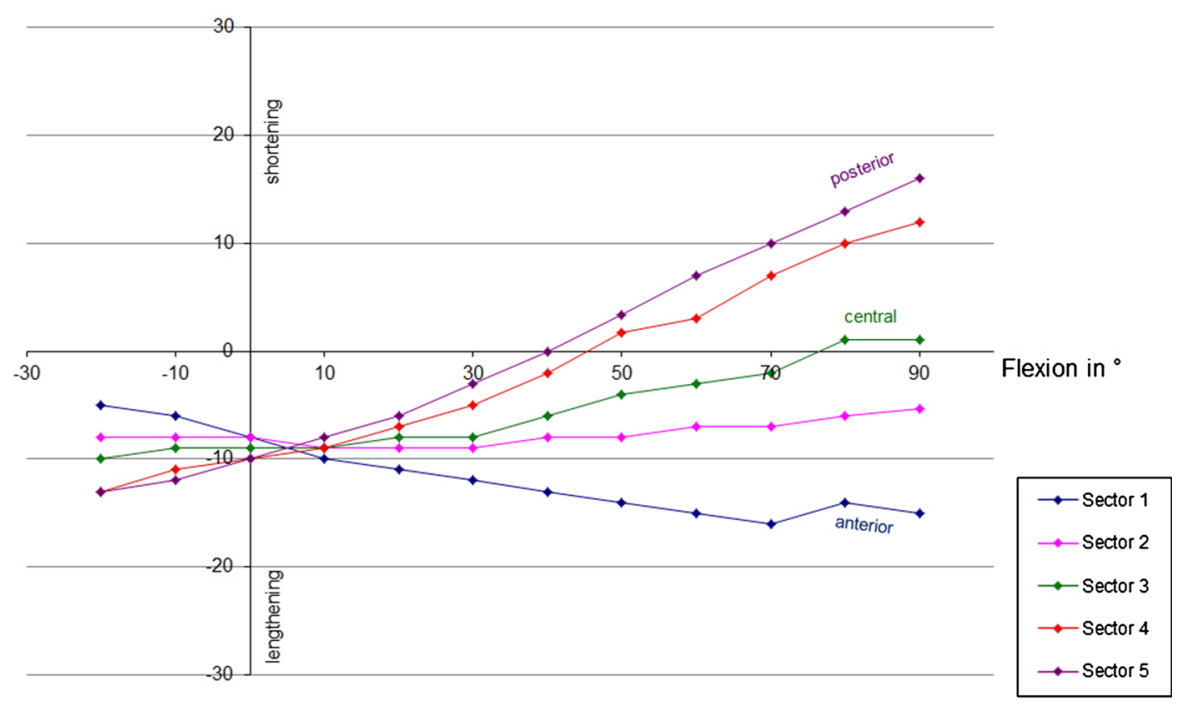

C Excursion in $\mathrm{mm}$

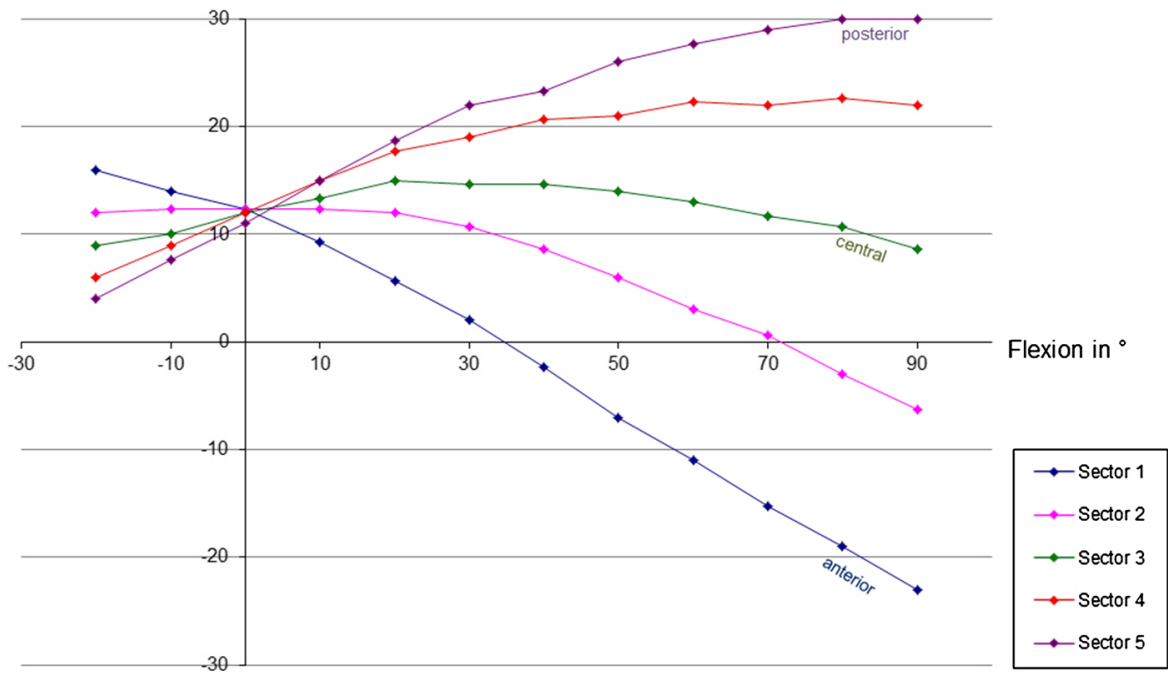


Table 3 Change of muscle excursion in percent at $70^{\circ}$ and $90^{\circ}$ of hip flexion for the proximalized (P) and distalized (D) trochanter for sectors 1,3 and 5

\begin{tabular}{|c|c|c|c|c|c|c|}
\hline \multirow[t]{2}{*}{ Hip flexion $\left(^{\circ}\right)$} & \multicolumn{2}{|l|}{ S1 } & \multicolumn{2}{|l|}{ S3 } & \multicolumn{2}{|l|}{ S5 } \\
\hline & $\mathrm{P}$ & $\mathrm{D}$ & $\mathrm{P}$ & $\mathrm{D}$ & $\mathrm{P}$ & $\mathrm{D}$ \\
\hline 70 & -68 & +13 & +360 & -89 & +13 & +1 \\
\hline 90 & -72 & +45 & +433 & +10 & +32 & -4 \\
\hline
\end{tabular}

A negative value indicates decrease and a positive value increase of excursion. In sector 3, the excursions are small (isometric sector), therefore already small changes lead to high values

extension from the flexed position, i.e. during stair climbing or while getting up from a chair. In addition, the movement of the insertion in the sagittal plane is least at this position.

By transferring the greater trochanter, this balance is disturbed. In particular, the distalization of the greater trochanter results that only the most anterior part of the muscle (sector 1) will shorten during flexion. The isometric sectors move anteriorly, and the central sector is, functionally, added to the posterior sectors. Theoretically, this increases strength for climbing stairs or getting up from a chair but it is most likely neutralized by the $69 \%$ decrease in the lever arm of the posterior sectors. Distalization of the trochanter not only changes the balance between active and passive muscle sectors but also changes the amount of excursion of the muscle fibers. Distalization of the greater trochanter by $10 \mathrm{~mm}$ will also lead to a $10 \%$ increase in the muscle fiber length of the gluteus medius. It has been shown that a $10 \%$ increase of sarcomere length from resting length will lead to a decrease in tension generation by approximately $5 \%$ [4]. Theoretically this can be compensated by an increase of the sarcomeres. Increase of serial sarcomere numbers was observed in an animal model after muscle lengthening [9] but in humans, the increase did not restore muscle mechanics in the stretched forearm muscles with loss of muscle force [21]. Maximum tetanic tension was significantly reduced. Applied to the abductor muscles this could indicate that distalization of the greater trochanter with stretching of gluteus medius would increase passive tension but also a significant loss of muscle strength.

Proximalization of the greater trochanter has a different impact on function. The isometric muscle area moves anteriorly, leading to an imbalance in favor of the posterior muscle sectors. For extension, more sectors are recruited which together with the $30 \%$ increase of the lever arm for extension leads to an increase of strength for extension, at the expense for flexion strength (less active muscle sectors, $40 \%$ shorter anterior lever arm). Overall, extension strength (from $30^{\circ}$ and $60^{\circ}$ flexion to extension) was improved substantially with proximalization of the trochanter. This is rather surprising, because the general believe was that proximalization of the trochanter always lead to weakening of the hip abductors which is probably true for level walking but not for climbing stairs.

For isolated abduction, both distalization and proximalization result in longer excursions. Therefore, a shift from isometric to isotonic muscle action is observed, which indicated longer muscular contraction and relaxation, which in turn causes more muscular work. This could explain earlier fatigue of the abductor muscles during walking. Although isolated abduction is rare and is most often combined with variable amounts of flexion, these findings indicate that the work and force needed to walk
Fig. 4 Excursion of the muscle fibers for rotation. Positive values on the $x$ axis correspond to internal and negative values to external rotation

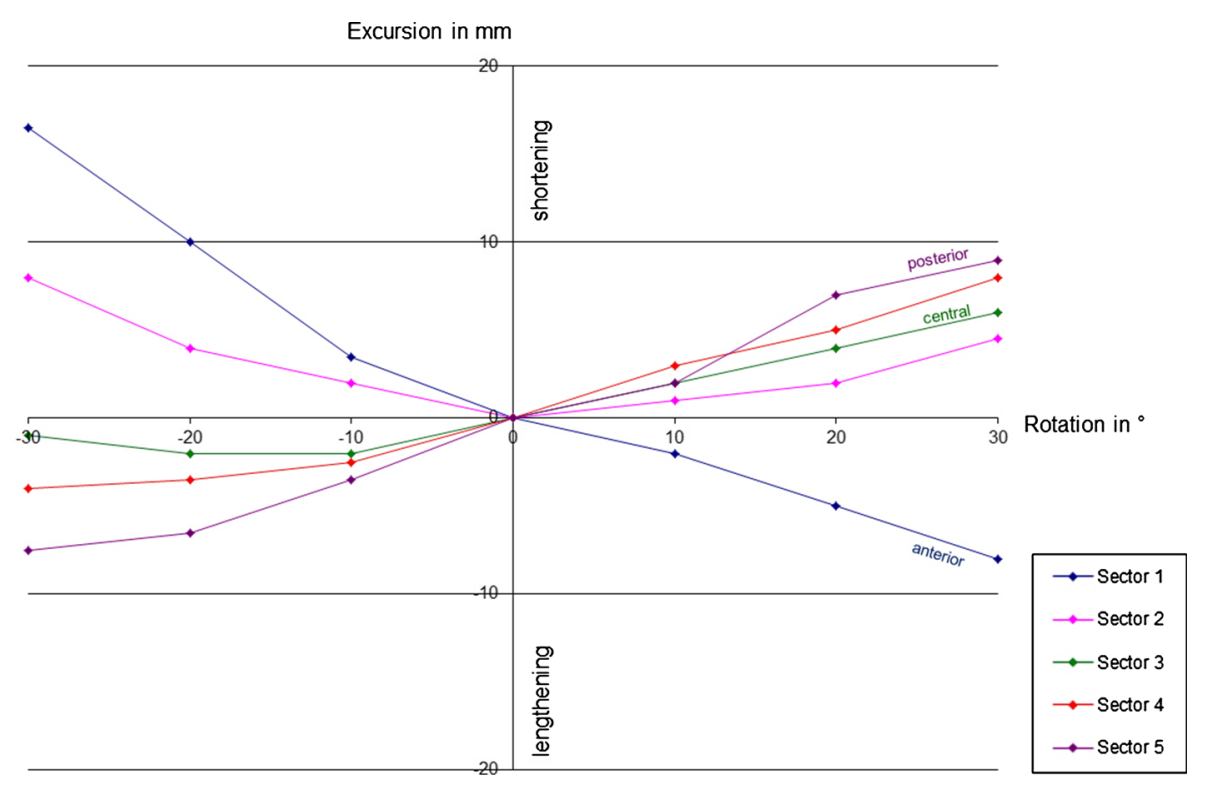


a

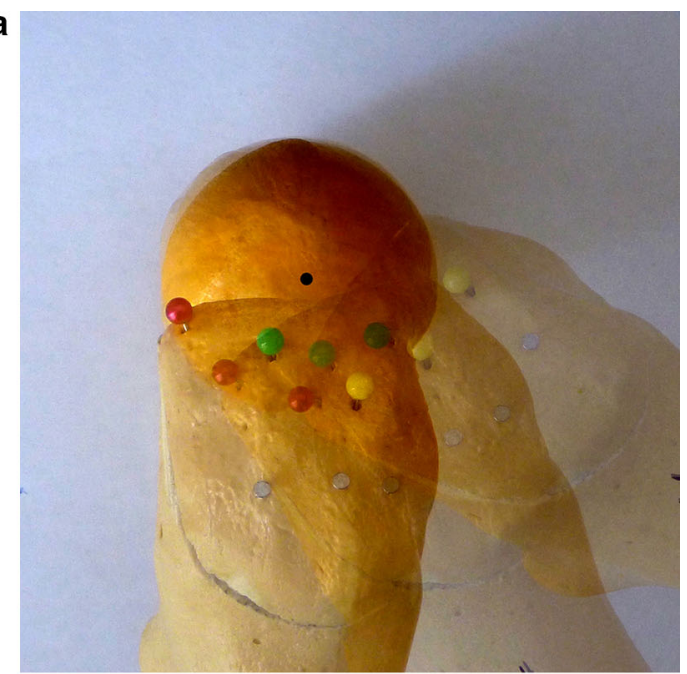

b

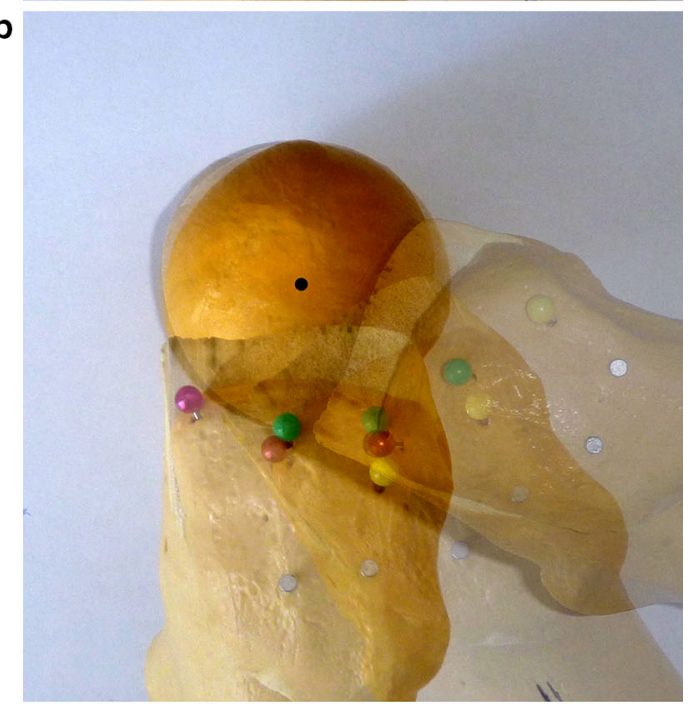

C

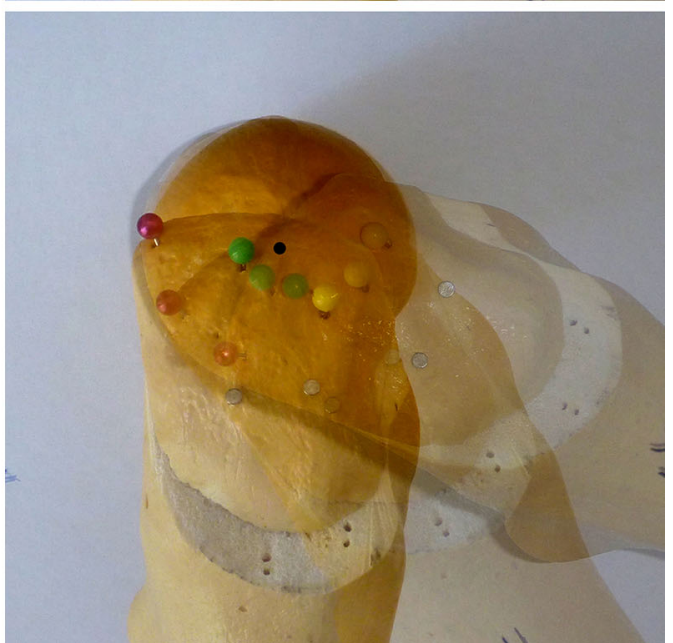

increase substantially, more for the distalized than for the proximalized trochanter, and may explain fatigue and subsequent pain in the trochanter area.

The string model has been used previously and was validated in a cadaver-based model [2]. Nevertheless, there
4Fig. 5 a Position of insertion of sector 5 (red), sector 3 (green), and sector 1 (yellow) at $0^{\circ}, 30^{\circ}$, and $60^{\circ}$ of hip flexion with the trochanter at the anatomic position. Black point center of rotation of femoral head. b Position of insertion of sector 5 (red), sector 3 (green), and sector 1 (yellow) at $0^{\circ}, 30^{\circ}$, and $70^{\circ}$ of hip flexion with the trochanter at the distalized position. Compared to a, the distance between the insertions is increased. Black point center of rotation of femoral head. c Position of insertion of sector 5 (red), sector 3 (green), and sector 1 (yellow) at $0^{\circ}, 30^{\circ}$, and $70^{\circ}$ of hip flexion with the trochanter at the proximalized position. Compared to a, the distance between the insertions is decreased. Black point center of rotation of femoral head

Table 4 Comparison of lever arms of sectors 1,3 , and 5 in $0^{\circ}, 30^{\circ}$, and $60^{\circ}$ of flexion for normal $(\mathrm{N})$, proximalized $(\mathrm{P})$, and distalized (D) trochanters

\begin{tabular}{|c|c|c|c|}
\hline & $\begin{array}{l}\text { Anatomic } \\
(\mathrm{mm})\end{array}$ & $\begin{array}{l}\text { Distalized } \\
(\mathrm{mm})\end{array}$ & $\begin{array}{l}\text { Proximalized } \\
(\mathrm{mm})\end{array}$ \\
\hline \multicolumn{4}{|c|}{ Sector 1} \\
\hline $0^{\circ}$ & 16 & $24(+50 \%)$ & $11(-32 \%)$ \\
\hline $30^{\circ}$ & 24 & $37(+42 \%)$ & $15(-32 \%)$ \\
\hline $60^{\circ}$ & 26 & $43(+65 \%)$ & $15(-42 \%)$ \\
\hline \multicolumn{4}{|c|}{ Sector 3} \\
\hline $0^{\circ}$ & -7 & $-3(-58 \%)$ & -7 \\
\hline $30^{\circ}$ & 3 & $13(+433 \%)$ & $-4^{\mathrm{a}}$ \\
\hline $60^{\circ}$ & 13 & $28(+215 \%)$ & $3(-77 \%)$ \\
\hline \multicolumn{4}{|c|}{ Sector 5} \\
\hline $0^{\circ}$ & -25 & $-27(+8 \%)$ & $-22(-12 \%)$ \\
\hline $30^{\circ}$ & -20 & $-15(-25 \%)$ & $-23(+15 \%)$ \\
\hline $60^{\circ}$ & -9 & $3^{\mathrm{a}}$ & $-17(+90 \%)$ \\
\hline
\end{tabular}

Negative values indicate position posterior to the center of rotation. The axis of action was inclined $20^{\circ}$ posteriorly for sector $5,0^{\circ}$ for the central sector 3 and $20^{\circ}$ anteriorly for sector 1

${ }^{\text {a }}$ Inversion of lever arm to the opposite function (extension to flexion)

are several limitations related to the use of a string model. First, strings represent only parts of the muscle. We attempted to route each string in the center of each sector and, while this does not necessary represent the action of the peripheral fibers of a sector, it gives an average of the function of a sector. In order to keep the system simple, we restricted the model to five sectors. Only passive excursions of the muscle fibers were measured. Shortening was attributed to contraction, and elongation was ascribed to the ability to resist a movement. However, we do not know how and when the different parts of the muscle are activated, if muscle sectors are counterbalancing or supporting each other. Unfortunately, this currently is the only way to get this information, as it is impossible to perform a study examining active muscle contraction at this anatomic level in vivo.

Another limitation is that we did not assess lateralization of the trochanter in this study, although it has been reported that lateralization is the more important component of any 
reconstruction $[15,24]$. Whether this is the case has been debated in the literature [17], but we did not expect major alterations of muscle mechanics. From a theoretical point of view, lateralization should not affect the balance of muscle excursion but increases the lever arm for abduction movements. In addition, the study was conducted on hips assuming normal anatomy. While the principal mechanical impact may also be applied to the diseased hip, it only can be speculated whether is applies also to situations with pathological soft tissues (atrophy, contractures, etc.).

In addition, it must be pointed out that the conclusions and considerations are based on an experimental lab setting and transfer to clinical practice may not necessarily apply.

Overall, both proximal and distal advancement of the greater trochanter have an impact on muscle balance, with transfer of the isometric muscle sector posteriorly and anteriorly, respectively. This dysbalance, together with unfavorable change of lever arm, loss of strength due to stretching (distalization) and shortening (proximalization) of the muscle and increased need of energy due to longer contraction amplitudes, may explain earlier fatiguing of the abductor musculature. Optimal balancing and excursion of the muscle is when the tip of the greater trochanter is at level with the hip rotation center. In hips with high riding trochanter, excessive distalization should be avoided.

Conflict of interest The authors certify that they have no commercial association that might pose a conflict of interest in connection with the submitted article.

Ethical standard The authors certify that his or her institution has approved the human protocol for this investigation and that all investigations were conducted in conformity with ethical principles of research.

\section{References}

1. Anderson LA, Erickson JA, Severson EP, Peters CL (2010) Sequelae of Perthes disease: treatment with surgical hip dislocation and relative femoral neck lengthening. J Pediatr Orthop 30:758-766

2. Beck M, Sledge JB, Gautier E, Dora CF, Ganz R (2000) The anatomy and function of the gluteus minimus muscle. $\mathrm{J}$ Bone Joint Surg Br 82-B:358-363

3. Chippinddale A, Haddad F, Galante J, Muirhead-Allwood S, Chao E, Stoller DW (2000) Interactive hip. CD-ROM. Primal Pictures Ltd., London

4. Edman KA, Reggiani C (1987) The sarcomere length-tension relation determined in short segments of intact muscle fibres of the frog. J Physiol 385:709-732

5. Eftekhar NS (1976) Dislocation and instability complicating low friction arthroplasty of the hip joint. Clin Orthop Relat Res 121:120-125
6. Espinosa N, Beck M, Rothenfluh DA, Ganz R, Leunig M (2007) Treatment of femoro-acetabular impingement: preliminary results of labral refixation. Surgical technique. J Bone Joint Surg Am 89(Suppl 2 Pt.1):36-53

7. Fackler CD, Poss R (1980) Dislocation in total hip arthroplasties. Clin Orthop Relat Res 151:169-178

8. Flack NA, Nicholson HD, Woodley SJ (2014) The anatomy of the hip abductor muscles. Clin Anat 27:241-253

9. Friden J, Ponten E, Lieber RL (2000) Effect of muscle tension during tendon transfer on sarcomerogenesis in a rabbit model. J Hand Surg Am 25:138-143

10. Free SA, Delp SL (1996) Trochanteric transfer in total hip replacement: effects on the moment arms and force-generating capacities of the hip abductors. J Orthop Res 14:245-250

11. Ganz R, Gill TJ, Gautier E, Ganz K, Krügel N, Berlemann U (2001) Surgical dislocation of the adult hip a technique with full access to the femoral head and acetabulum without the risk of avascular necrosis. J Bone Joint Surg Br 83:1119-1124

12. Ganz R, Huff TW, Leunig M (2009) Extended retinacular softtissue flap for intra-articular hip surgery: surgical technique, indications, and results of application. Instr Course Lect 58:241-255

13. Goodwin RC, Mahar A, Wedemeyer M, Wenger D (2007) Abductor length alterations in hips with SCFE deformity. Clin Orthop Relat Res 454:163-168

14. Kaplan SJ, Thomas WH, Poss R (1987) Trochanteric advancement for recurrent dislocation after total hip arthroplasty. J Arthroplasty 2:119-124

15. Kelikian AS, Tachdjian MO, Askew MJ, Jasty M (1983) Greater trochanteric advancement of the proximal femur: a clinical and biomechanical study. Hip 77-105

16. Leunig M, Ganz R (2011) Relative neck lengthening and intracapital osteotomy for severe Perthes and Perthes-like deformities. Bull NYU Hosp Jt Dis 69(Suppl 1):S62-S67

17. Macnicol MF, Makris D (1991) Distal transfer of the greater trochanter. J Bone Joint Surg Br 73:838-841

18. Pucher A, Ruszkowski K, Bernardczyk K, Nowicki J (2000) The value of distal greater trochanteric transfer in the treatment of deformity of the proximal femur owing to avascular necrosis. J Pediatr Orthop 20:311-316

19. Sobotta J (1988) Atlas der Anatomie des Menschen. Band 2, 19. Auflage. Staubesand J (ed.) Urban\&Schwarzenberg, pp 302-311

20. Schünke M, Schultee E, Schumacher U. (2005) Prometheus. Lernatlas der Anatomie. Allgemeine Anatomie und Bewegunssystem. Georg Thieme Verlag. 2.3 Hüft- und Gesässmuskulatur: äussere Hüftmuskeln 2.3:424-425

21. Takahashi M, Ward SR, Friden J, Lieber RL (2012) Muscle excursion does not correlate with increased serial sarcomere number after muscle adaptation to stretched tendon transfer. J Orthop Res 30:1774-1780

22. Tauber C, Ganel A, Horoszowski H, Farine I (1980) Distal transfer of the greater trochanter in cox vara. Acta Orthop Scand 51:661-666

23. Theivendran K, Hart WJ (2009) Is the tip of the greater trochanter a reliable reference for the rotation centre of the femoral head in total hip arthroplasty? Acta Orthop Belg 75:472-476

24. Wagner H (1978) Transplantation of the greater trochanter. In: Weil UH, Dega W, Macewen GD (eds) Acetabular dysplasia: skeletal dysplasias in childhood. Springer, Berlin, pp 99-102

25. Williams PL (ed) (1995) Gluteus medius. In: Gray's anatomy, 38th edn. Churchill Livingstone, London 